\title{
Commentary: Mutation: source of variation in evolutionary ecology
}

\author{
Charles B. Fenster ${ }^{1}$ (D) . Courtney J. Murren ${ }^{2}$ (D)
}

Received: 16 April 2020 / Accepted: 4 May 2020 / Published online: 17 May 2020

(c) Springer Nature Switzerland AG 2020

\begin{abstract}
Mutation is a key force in evolutionary ecology. While the source of new genetic variation, mutation is often considered separately from other mechanisms. This commentary accompanies the mini-special issue/topical collection to draw attention to mutation in evolutionary ecology. We highlight each of the diverse papers, and provide context for future studies. New technologies and datasets coming online suggest a vibrant future of mutation investigations in evolutionary ecology.
\end{abstract}

Keywords Mutation · Evolutionary ecology $\cdot$ Virus $\cdot$ E. coli $\cdot$ Arabidopsis $\cdot$ Adaptive substitution

Mutation is often referred to as "the ultimate source of new variation" on which selection acts, yet mutation is not often the centerpiece of adaptive evolutionary considerations. This perspective of the limited role of mutation to selection response dates back to early literature viewing organisms as optimally adapted to their environment, thus mutations were thought to be a perturbation to the phenotype and hence deleterious (Timoféeff-Ressovsky 1940; Muller 1950). However, simultaneously and early in the neo-Darwinian synthesis, Fisher (1930) made explicit the notion that populations may not be at their adaptive optimum and modeled the evolution of a genotype through mutation as a walk through fitness landscape space. These perspectives have themselves undergone interesting intellectual evolution with the former argument becoming the foundation of the neutral (Kimura 1968) or nearly neutral theory (Ohta 1973) of molecular evolution where DNA substitution reflects drift processes with counter evidence provided by McDonald-Kreitman (1991) tests and their derivatives demonstrating adaptive DNA substitution. These contrasting notions of the contribution of mutation to evolutionary process are complicated by the biological reality that life-processes are rarely monolithic, but are contingent on context. In the case of mutations, this context has two components the environment in which the phenotype

Courtney J. Murren

murrenc@cofc.edu

Charles B. Fenster

Charles.Fenster@sdstate.edu

1 Oak Lake Field Station, South Dakota State University, Brookings, SD 57007, USA

2 Department of Biology, College of Charleston, Charleston, SC 29424, USA 
is found as well as the genomic environment, i.e., the particular sequence that mutates. Furthermore, the balance between selection and drift is in turn influenced by demographic history.

Now with advances in genomics, molecular genetics and computational approaches, our ability to detect and evaluate the fitness effects of mutation is growing at a rapid rate. Such advances have demonstrated that mutation rates are variable by species, populations, areas of the chromosome and across different ecological environments. Data on distributions of mutational effects on phenotypes, particularly fitness phenotypes, are further enhanced by computational advances and taking these problems into ecological settings. In this issue are four contributions that bring together ideas from theoretical, molecular, computational and various experimental approaches across many organisms and systems to improve our understanding of the contributions of mutation to evolutionary ecology.

To draw attention to the role of mutation in evolutionary ecology, the articles in this mini-special issue/topical collection bring together distinct perspectives on mutation biology. The articles, described below, highlight theoretical, microbial, viral and plant approaches to expanding our knowledge on this central mechanism of evolution.

Moutinho et al. (2020), "Variation of the adaptive substitution rate between species and within genomes," summarize the current state-of-the-art of theoretical population genetic development, statistical approaches and computational methods to quantify the distribution of fitness effects of new mutations and review the factors that affect the molecular rate of adaptation. They provide "a comprehensive review of methods used to infer the molecular adaptive rate, the potential drivers of adaptive evolution and how positive selection shapes molecular evolution within genes, across genes within species and between species." These methods now incorporate both population genetic and phylogenetic approaches to quantify mutation effect on fitness. Summarizing the literature they suggest that the contribution of effective population size is primarily to promote the purging of deleterious mutations while at the genome scale, molecular rates of adaptation are consistent with models examining linked selection, i.e., factors such as gene density, recombination and mutation rate. Their recommendations for future work offer promise of fruitful avenues of modeling work in evolutionary ecology and mutation.

Caudill et al. (2020), "CpG-creating Mutations are Costly in Many Human Viruses" demonstrate that yet an additional class of deleterious mutations, in addition to nonsynonymous and indels in coding regions exist. CpG sites are rare in eukaryotic and HIV genomes. For the eukaryotes, it is thought that $\mathrm{CpG}$ sites are rare because they are prone to mutation when methylated, however less is known about whether $\mathrm{CpG}$ sites are rare across virus taxa. After analyzing the allele frequencies of $\mathrm{CpG}$-creating and non-CpG-creating mutations across various strains, subtypes, and genes of viruses using existing data obtained from Genbank, HIV Databases, and Virus Pathogen Resource it is clear that CpG sites are generally rare in viral genomes and thus likely costly for viruses. Future research is needed on why CpG sites are costly to viruses. Growing publically available sequence data offer opportunities for cross system comparisons.

Rutter et al. (2020), "Disrupting the disruptors: the consequences of mutations in mobile elements for ecologically important life history trait" provides among the clearest evidence to date that transposable elements (TEs) can contribute to the distribution of quantitative trait expression, increasing or decreasing mean phenotype. Since TEs are often considered to be the manifestation of previous parasitization of the genome, they hypothesized that insertions within TEs would have a more positive effect on traits. Instead they provide robust results that mutants in transposable element genes were similar to mutants in other types of loci for the measured life history traits critical to 
population processes. Overall, Rutter et al.'s contribution should draw renewed attention to an overlooked and tremendously important component of the eukaryotic genome.

Finally, Clarke et al. (2020), "The effect of environmental heterogeneity on the fitness of abiotic resistance mutations in Escherichia coli." examine whether novel mutations conferring antibiotic resistance express genotype $\mathrm{x}$ environment interactions, namely whether in the absence of the antibiotic the mutation is costly to the bacteria. Estimating the fitness of six resistant mutants of Escherichia coli in media reflecting ecological niches, human hosts, and standard lab conditions, they found extensive evidence for genotype $\mathrm{x}$ environment interactions, with different genotypes favored in different environments. However, the results were not uniform in that resistance alleles did not suffer a cost in some environments, raising the possibility of environmental reservoirs of resistance. Their findings have several implications. With regards to resistance, it is clearly necessary to consider the environment in which these microbes are found and whether they are conducive to the maintenance of resistant genotypes. More broadly, their findings indicate that no easy generalization can be made regarding how environment modulates the expression of mutations, while laying the groundwork for expansion of the study of these questions.

\section{Conclusions and future directions}

It is an exciting time for mutation research. With access to new molecular genetic, ecological, and computational tools and resources, additional opportunities for the study of mutation in the context of evolutionary ecological biology are here. Even as recently as a decade ago, tools and sequence data were largely restricted to model organisms. These tools have been expanded and have been leveraged to study agricultural and wild species. These advances have enabled a growing library of questions in evolutionary ecology to be addressed more fully and in finer detail than ever before. Integrating empirically grounded mutation rates, and variation in fitness effects of mutation into models and hypotheses, will expand on these successes further still. Yet challenges lie ahead. With increasingly large and complex datasets, development of new computational tools that are effective for use across clades are needed. Experiments to test questions on the impacts of mutation in ecologically relevant environments will require large efforts and new quantitative approaches. These challenges highlight the need for diverse training of future evolutionary and ecological scientists at undergraduates and graduate levels, and in particular in the data sciences so that the next cadre are just as facile in the creation of pipelines as students are now with the explosion of new statistical approaches.

In summary, these papers provide the conceptual and experimental frameworks to understand further the contribution of mutations to evolutionary process. Overall, it is obvious that there are diverse open avenues for research in mutational biology. These are exciting opportunities given technological advances and global datasets in ecology and genomics to bring together, ecologists, evolutionists, geneticists and computational biologists and synthesize data from across systems.

Acknowledgements We thank the authors, reviewers and editors for their contributions to this special issue/ topical collection. C. Fenster's work on mutations has been supported by NSF. C. Murren's work on mutations has been supported by NSF and USDA. 


\section{Compliance with ethical standards}

Conflict of interest The authors declare no conflict of interest.

\section{References}

Caudill V, Qin S, Winstead R, Kaur J, Tisthammer, K, Pineda EG, Solis C, Cobey S, Bedford T, Carha O, Eggo RM, Koelle K, Lythgoe K, Regoes R, Roy S, Allen N, Aviles M, Baker BA, Bauer W, Bermudez S, Carlson C, Castellanos E, Catalan FL, Chemel AK, Elliot J, Evans D, Fiutek N, Fryer E, Goodfellow SA, Hecht M, Hopp K, Hopson Jr ED, Jaberi A, Kinney C, Lao D, Le A, Lo J, Lopez AG, Lopez A, Lorenzo FG, Luu GT, Mahoney AR, Melton RL, Nacimiento GD, Prahananga A, Rodriques NS, Shieh A, Sims J, Singh R, Sulaeman H, Thu R, Tran K, Tran L, Winters EJ, Wong A, Pennings PS (2020) CpG-creating mutations are costly in many human viruses. Evolut Ecol. 34(3):339-359. https:// doi.org/10.1007/s10682-020-10039-z

Clarke L, Pelin A, Phan M, Wong A (2020) The effect of environmental heterogeneity on the fitness of abiotic resitstance mutation in Escherichia coli. Evolut Ecol. 34(3):379-390. https://doi.org/10.1007/ s10682-019-10027-y

Fisher RA (1930) The genetical theory of natural selection. Oxford Univ. Press, Oxford

Kimura M (1968) Evolutionary rate at the molecular level. Nature 217:624-626

McDonald J, Kreitman M (1991) Adaptive evolution at the Adh locus in Drosophila. Nature 351:652-654

Moutinho AF, Bataillon T, Dutheil JY (2020) Variation of the adaptive substitution rate between species and within genomes. Evolut Ecol. 34(3):315-338. https://doi.org/10.1007/s10682-019-10026-Z

Muller HJ (1950) Our load of mutations. Am J Human Genet 2:111

Ohta T (1973) Slightly deleterious mutant substitutions in evolution. Nature 246:96-98

Timoféeff-Ressovsky NW (1940) Mutations and geographical variation. In: Huxley J (ed) The new systematics. Oxford University Press, Oxford, pp 73-136

Rutter MT, Bisner, AB, Kohler C, Morgan K, Musselman O, Pickel J, Tan J, Yamasaki Y, Willson J, Callahan HS, Strand AE, Murren CJ (2020) Disrupting the disruptors: the consequences of mutations in mobile elements for ecologically important life history trait. Evolut Ecol. 34(3):363-377. https://doi. org/10.1007/s10682-020-10038-0

Publisher's Note Springer Nature remains neutral with regard to jurisdictional claims in published maps and institutional affiliations. 